\title{
A computational model of associative learning and chemotaxis in the nematode worm C. elegans
}

\author{
Peter A Appleby, Netta Cohen ${ }^{*}$ \\ From Nineteenth Annual Computational Neuroscience Meeting: CNS*2010 \\ San Antonio, TX, USA. 24-30 July 2010
}

The nematode worm C. elegans is an exciting model system for experimentalists and modelers alike. It has a relatively small nervous system, made up of just 302 neurons in the adult hermaphrodite, that has been mapped in detail using serial section electron microscopy. Despite the simplicity of its nervous system $C$. elegans displays a range of interesting behaviors. This includes thermo- and chemotaxis and the modulation of locomotion strategies in response to the presence of food. In chemotaxis C. elegans will move up or down a chemical gradient dependent on whether the chemical acts as an attractant or repellent. It does this in two distinct ways, first by gradually steering left or right until the worm points up or down the gradient and second by modulating the probability of initiating a sharp series of turns (known as a piroeutte) along with the final orientation of the worm after the pirouette has finished. Experimental work has shown that the chemotaxis response is dynamic and that the degree of influence a particular chemical has on navigation can be changed, or even reversed depending on experience. Changes are reversible, specific to the chemical in question, and can be generated by classical conditioning experiments. All of these are hallmarks of associative learning, a sophisticated process that requires integration of multiple signals to produce a coordinated change in a behavioral response.

Despite the wealth of experimental data on learning in chemotaxis in C. elegans, comparatively little is known about how the known circuitry of $C$. elegans carries out the computations that underlie it. Even less is known about how that circuitry changes during

\footnotetext{
* Correspondence: n.cohen@leeds.ac.uk

School of Computing, University of Leeds, Leeds LS2 9JT, UK
}

associative learning. Here, we focus on the worm's chemotaxis and the ability of C. elegans to learn associations between salt $(\mathrm{NaCl})$ concentrations and food. We draw upon existing experimental data from a variety of sources including electrophysiological and anatomical data to construct a simplified $\mathrm{NaCl}$ chemotaxis circuit in C. elegans. This circuit consists of the left and right ASE amphid sensory neurons, which comprise the dominant $\mathrm{NaCl}$ sensation pathway in C. elegans, eight pairs of interneurons, and ten head motor neurons. Where possible the properties of individual neurons are constrained by electrophysiological and calcium imaging data.

We next define a set of experimentally observed behaviors we wish to reproduce, including gentle turning, modulation of pirouette frequency, control of final orientation following a pirouette, and associative learning. In particular, we are interested in the alteration in behavioral response to $\mathrm{NaCl}$ that arises due to the pairing of high concentrations of $\mathrm{NaCl}$ with food or starvation. We use this to derive a family of model networks with specific synaptic polarities, time scales of neuronal responses, and intrinsic neuronal properties that have the capacity to generate the specified set of behaviors. We implement one of these models and record the behaviour of model worms that are placed in a variety of simulated environments. We observe qualitatively realistic chemotaxis behavior and adaptation and demonstrate that our model is robust and tolerant to noise.

Our proposed chemotaxis circuit leads to a number of distinct predictions that could be used to test the model experimentally. This includes postulating the computational role of each neuron in the network and the locus and nature of the plasticity underpinning the 
experimentally observed associative learning. Our model also suggests that this plasticity be expressed not by changes in synaptic strength but by changes in the sensory neurons themselves. Thus, contrary to the prevailing view in the C. elegans community, plasticity in our model of chemotaxis is expressed at a neuronal rather than synaptic level.

C. elegans offers a unique opportunity to push the boundaries of systems neuroscience. The ability to model a neuronal circuit in such detail is a remarkable opportunity to study associative learning in an animal displaying a sophisticated set of behaviors and nontrivial learning. A biologically grounded model of behavior and learning in C. elegans has great potential to offer detailed and integrated understanding of sensory processing, synaptic plasticity and associative learning. Lessons learned from such models can be applied to other sensory and sensorimotor modalities in the worm with the eventual goal of producing an integrated model of the worm's sensorimotor system. We believe that theoretical insights gained from this endeavour will be invaluable in our study of larger, more complex nervous systems.

Published: 20 July 2010

doi:10.1186/1471-2202-11-S1-08

Cite this article as: Appleby and Cohen: A computational model of associative learning and chemotaxis in the nematode worm C. elegans. BMC Neuroscience 2010 11(Suppl 1):O8.
Submit your next manuscript to BioMed Central and take full advantage of:

- Convenient online submission

- Thorough peer review

- No space constraints or color figure charges

- Immediate publication on acceptance

- Inclusion in PubMed, CAS, Scopus and Google Scholar

- Research which is freely available for redistribution

Submit your manuscript at www.biomedcentral.com/submit 